\title{
Investigating neurophysiological correlates of metacontrast masking with magnetoencephalography
}

\author{
Sandra I. van Aalderen-Smeets ${ }^{1,2}$, Robert Oostenveld ${ }^{1}$, and Jens Schwarzbach ${ }^{1,2}$ \\ ${ }^{1}$ FC Donders Centre for Cognitive Neuroimaging, Nijmegen, the Netherlands \\ ${ }^{2}$ Faculty of Psychology, Department of Cognitive Neuroscience, Maastricht University, the Netherlands
}

Received 26.04.2005

Accepted 15.11.2005

\section{Keywords}

Metacontrast masking, MEG, object visibility, working memory

\section{ABSTRACT}

Early components of visual evoked potentials (VEP) in EEG seem to be unaffected by target visibility in visual masking studies. Bridgeman's reanalysis of Jeffreys and Musselwhite's (1986) data suggests that a later visual component in the VEP, around 250 ms reflects the perceptual effect of masking. We challenge this view on the ground that temporal interactions between targets and masks unrelated to stimulus visibility could account for Bridgeman's observation of a U-shaped time course in VEP amplitudes for this later component. In an MEG experiment of metacontrast masking with variable stimulus onset asynchrony, we introduce a proper control, a pseudo mask. In contrast to an effective mask, the pseudomask should produce neither behavioral masking nor amplitude modulations of late
VEPs. Our results show that effective masks produced a strong U-shaped perceptual effect of target visibility while performance remained virtually perfect when a pseudomask was used. The visual components around $250 \mathrm{~ms}$ after target onset did not show a distinction between mask and pseudomask conditions. The results indicate that these visual evoked potentials do not reveal neurophysiological correlates of stimulus visibility but rather reflect dynamic interactions between superimposed potentials elicited by stimuli in close temporal proximity.

However, we observed a postperceptual component around $\mathbf{3 4 0} \mathbf{~ m s}$ after target onset, located over temporal-parietal cortex, which shows a clear effect of visibility. Based on P300 ERP literature, this finding could indicate that working memory related processes contribute to metacontrast masking.

\section{INTRODUCTION}

Visual backward masking refers to the reduced visibility of a visual target stimulus in the presence of a second stimulus, the mask, presented later in time. Presenting different types of mask stimuli results in different behavioral masking functions, that is, functions relating the visibility of the target to the length of the interval between the target and mask. A pattern or noise mask usually results in monotonically increasing visibility of the target stimulus (Breitmeyer, 1984). Metacontrast masking refers to conditions in which the inner contours of a mask stimulus do not spatially overlap with those of a preceding target stimulus (Breitmeyer \& Ogmen, 2000). For certain durations of the stimuli metacontrast masking results in a non-monotonic $\mathrm{U}$-shaped dependence of target visibility on Stimulus Onset Asynchrony (SOA) between target and mask (Breitmeyer, 1984).

Correspondence concerning this article should be addressed to Sandra van Aalderen-Smeets, FC Donders Centre, Kapittelweg 29, PO Box 9101, 6500 HB Nijmegen, The Netherlands, tel. +31243668065 , fax. +31243610989 . Electronic mail may be sent via Internet to Sandra.Smeets@fcdonders.ru.nl. 
Several hypotheses concerning the mechanisms of masking have been postulated (for a review see Breitmeyer \& Ogmen, 2000). The dual channel, sustained-transient theory of visual masking (Breitmeyer, 1984) postulates two hypothetical channels that are involved in the processing of a visual stimulus. A slow and sustained channel processes object features like brightness and color, while a faster transient channel processes coarse patterns and signals spatial location and motion of a stimulus. Accordingly, an interaction of these two channels is thought to give rise to metacontrast masking: The fast responding transient channel activated by the mask inhibits the slow responding sustained channel activated by the target, hence the target related activity is disrupted and the target is not processed any further.

Another theory to explain backward masking is based on recurrent processing (Bridgeman, 1980; Enns \& Di Lollo, 2000). A visual stimulus produces an early burst of activity in striate areas. After being carried to higher extrastriate visual areas through cortico-cortical connections the information returns to early areas. It is thought that the architecture of re-entry searches for a match between a descending code and the low-level activity. This recurrent, or iterative, processing lasts until a match is made or new information is entered into the system. Consequently, the same cells in striate cortex encode different information about the same stimulus at different times. Recurrent processing is assumed to be necessary to group object features attentively and to make them available for conscious perception (Lamme \& Roelfsema, 2000). In the case of metacontrast masking, activity related to the mask enters the early visual areas at the same time as target related information from recurrent loops gets back to these same areas. In that case, feedback information does not match the activity from the first burst of activity, and the target stimulus will not be consciously perceived.

Neurophysiological correlates related to the processing of masking stimuli can shed light on how processing of the target and mask stimulus evolves over time in the brain. They provide information about the latencies at which the processing of the target is disrupted or modulated. Previous studies have searched for physiological markers of masking using electroencephalography (EEG). This technique allows one to pinpoint the latency at which the target related brain activity is modulated by brain responses triggered by the mask.

The motivation for this present experiment is based on an EEG study by Jeffreys and Musselwhite (1986) and the reanalysis of their data by Bridgeman (1988). Jeffreys and Musselwhite investigated whether the analysis of the target stimulus is suppressed at a pre-cortical or early cortical level, as the dual channel approach predicts (Jeffreys \& Musselwhite, 1986). They tested whether metacontrast related inhibition or suppression is reflected in early visual evoked potentials (VEPs), namely the C1 and C2 component. Scalp distributions of $\mathrm{C} 1$ and $\mathrm{C} 2$ reflect the respective sites of origin in the striate and extrastriate visual cortex (Jeffreys, 1971; Jeffreys \& Axford, 1972). They found no effect of metacontrast masking in the C1 or C2 amplitude when using either a single target flanked by four masks or multiple targets flanked by multiple masks spanning half of the visual field. However, both stimulus arrays did yield a clear U-shaped masking function in a separate psychophysical study. These results are supported by an earlier EEG study of Schiller and Chorover (1966), which also did not find a modulation of early VEP components by metacontrast masking.

The findings of Jeffreys and Musselwhite seem to rule out the early modulation of target related activity by the mask stimulus, but the authors did not address how their data might relate to alternative accounts of visual masking, for example, the influence of recurrent processes on later components of VEPs.

Bridgeman (1988) reanalyzed Jeffreys and Musselwhite's (1986) data to look for a modulation of the VEP at a later timepoint ( $250 \mathrm{~ms}$ ) after stimulus onset. A modulation around this latency has been found in single neuron activity in cat and monkey striate cortex (Bridgeman, 1975, 1980). Bridgeman measured the VEP amplitude at this latency for all SOAs and showed a $\mathrm{U}$-shaped modulation of the amplitude corresponding to the behavioral $\mathrm{U}$-shaped masking function. He interpreted these modulations as reflecting visual masking due to recurrent processing.

We doubt that Bridgeman's conclusion is justified because mere temporal interactions between target and mask could produce such a U-shape without being related to visibility. The time it takes to process a stimulus is longer than the actual stimulus duration. When two stimuli are presented in close succession, the processing of these stimuli in visual cortex will overlap in time and will not necessarily sum up in a linear fashion. Hence, the VEP following two stimuli that are presented in rapid succession reflects the processing of both stimuli. If the time between the two stimuli varies, it will result in different VEP waveforms. In addition, the brain activity that is measured using EEG on the outside of the head reflects a summation of the signals from different brain areas. Given the VEP on a single electrode, it is not possible to deduce how the different signals from different stimuli and different brain areas contributed to this VEP. 
We therefore introduce a critical control for this temporal overlap in the present experiment. In addition to a standard masking procedure, we also present the target followed by a pseudomask at each SOA. This pseudomask is as similar to the mask as possible but is designed in a way that it does not lead to the behavioral effect of masking. For each SOA, we can compare trials of similar temporal characteristics (target, SOA, and mask) but with different behavioral masking effects, that is, masking versus nonmasking. If the U-shaped modulation that Bridgeman observed in the VEP amplitude is related to visibility, then the masking condition, which leads to U-shaped behavioral data, should also yield a U-shaped modulation of the VEP, while the pseudomask condition should yield flat (high) performance and produce no comparable change in VEP amplitude. In addition, the control condition can be used to determine whether an SOA interaction reflects perceptual or non perceptual processes. More specifically, we use the activity differences associated with the different physical masks to verify that a particular brain activity pattern has a perceptual origin. If we find a main effect of mask type, we can conclude that the corresponding activity reflects a perceptual process. If we do not find such significant main effects of masking on the brain responses, we must conclude that these brain activities are unspecific with respect to the visual appearance of the stimulus, and that they reflect processes at a post-perceptual level.

We used whole-head magnetoenchephalography (MEG) to measure the visual evoked fields (VEF) induced by the stimuli. The main benefits of MEG over EEG are that it is more sensitive to superficial sources in the brain and that MEG data contain less noise. In addition, MEG has a larger number of sensors and the signals are less spatially distorted by the skull. Otherwise, MEG and EEG are expected to produce very similar results. This is particularly true for the VEFs that occur at the same latencies as the corresponding ERPs.

\section{SUBJECTS AND METHODS}

\section{Subjects}

Sixteen subjects (ages 19-38, mean 27 years, 11 women) took part in a behavioral practice session of 30 minutes (see Stimuli and Task section). Out of these subjects, 14 showed a $U$-shaped masking function in the masking condition and no masking in the pseudomask condition (more than $80 \%$ correct). These 14 subjects participated in a subsequent MEG experiment. All subjects were right handed and had normal or corrected to normal vision. The MEG protocol was approved by the a.

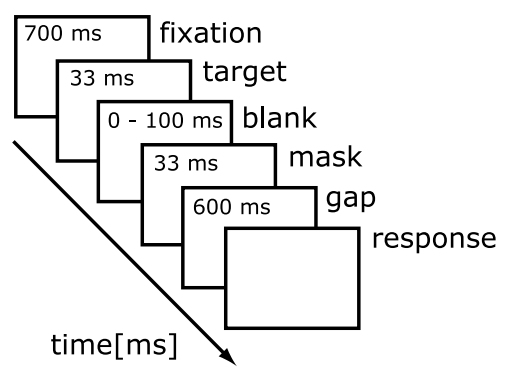

b.

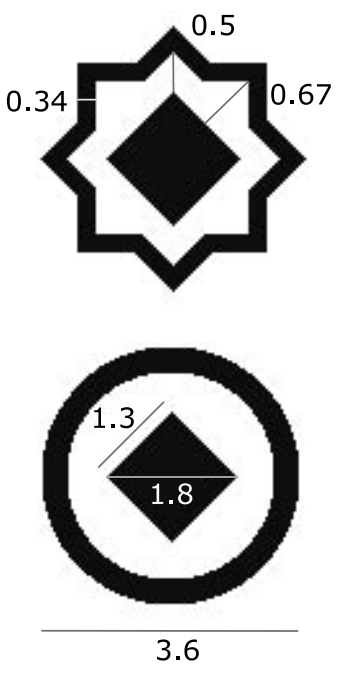

Figure 1. Stimulus Paradigm.

a. Schematic diagram of an example stimulus sequence. b. Size of the target and mask stimulus in degrees.

local ethics committee and all subjects gave written informed consent according to the Declaration of Helsinki.

\section{Stimuli and Task}

The stimulus set consisted of two target stimuli and two mask stimuli. The target stimulus was either a diamond or a square figure, size $1.3^{\circ}-1.8^{\circ}$, presented in the lower right quadrant of the visual field at an eccentricity of $6^{\circ}$ from a small fixation cross at the centre of the screen. All stimuli were black, had equal illuminance (4 Lux), and were projected on a light grey background (409 Lux). The two conditions in the experiment were defined by the type of mask used. The mask in the masking condition consisted of an overlapping diamond and square, size $3.6^{\circ}$, which formed a star shaped figure with a similar star shaped figure cut out of the middle (Figure $1 \mathrm{~b}$ ). The size of the mask was such that the inner contour of the mask was separated by $0.5-0.67^{\circ}$ from the outer contour of the target stimuli. The mask in the pseudomask condition was a ring shaped figure with similar sizes of the inner 
and outer contours as the star shaped figure. Because the contours of this mask do not share similar features with those of the target stimulus, the target should be less masked (Alpern, 1953; Breitmeyer, 1984).

Trials started with the presentation of a fixation cross, which stayed on the screen during the whole trial. After $700 \mathrm{~ms}$, a sequence of target and mask stimuli was presented on the screen (Figure $1 \mathrm{a}$ ). Both target and mask stimuli were presented for $33 \mathrm{~ms}$ with different SOAs (0, $33,50,66,83,133 \mathrm{~ms}$ ) between target and mask onset. At an SOA of $0 \mathrm{~ms}$, the target and the mask were presented simultaneously. At longer SOAs the mask followed the target. Subjects had to indicate which of the two targets, diamond or square, they had seen by pressing a button with their left or right index finger, respectively. The response had to be made within a time window of two seconds, starting $600 \mathrm{~ms}$ after offset of the mask stimulus. This $600 \mathrm{~ms}$ delay prevents contamination of the VEFs with activity from motor execution as well as an overestimation of target visibility (Lachter, Durgin, \& Washington, 2000). If subjects responded within these $600 \mathrm{~ms}$, a visual warning signal appeared and the trial was discarded from further analysis. After a response, the screen turned blank until the next trial started. Inter-trial intervals varied randomly with a range between 1,000 and $2,000 \mathrm{~ms}$ and a mean of $1,500 \mathrm{~ms}$.

A total of 28 conditions [ 6 SOAs $\times 2$ target types $\times$ 2 mask types, target presented alone (2 target types), mask presented alone ( 2 mask types)] were randomly presented with 40 repetitions each, resulting in 1,120 trials. The experiment was run in 8 blocks of 140 trials, each with a duration of about 8.5 minutes, depending on the subjects' response times. Subjects were free in deciding when to continue with the next block of trials.

\section{Procedure}

Visually evoked fields were recorded in a magnetically shielded room using a whole-head MEG system (Omega 2000, CTF Systems, Inc., Vancouver, Canada) with 151 axial gradiometers. The data were sampled at $600 \mathrm{~Hz}$ with a lowpass filter of $200 \mathrm{~Hz}$. Subjects were seated comfortably with their head located within the helmet of the MEG system and were instructed to move as little as possible during the whole experiment. The distance of the eyes to the screen was $68 \mathrm{~cm}$. Lights were turned off during the measurement. To measure eye movements and blinks, bipolar electrode pairs were placed lateral to the outer side of the left and the right canthus and above and below the right eye. Impedance of all electrodes was below $10 \mathrm{kOhms.}$ Prior to and after the MEG measurement the subject's head position relative to the gradiometer array was measured using coils positioned on the subject's nasion and at the bilateral external auditory meatus.

\section{Analysis \\ Behavioral data}

Behavioral responses were recorded during the MEG measurement. Trials in which subjects responded too early were discarded $(1.3 \%$ on average, range $0.3 \%-$ $-5.3 \%)$. The percentage correct of the responses was calculated for each SOA in both the masking and pseudomask condition, pooled over square and diamond target trials. A two-factorial repeated measures ANOVA was performed on the behavioral data of all subjects with the variables mask type and SOA.

\section{MEG data}

During preprocessing, all trials in which subjects responded too early were excluded. The EOG was visually inspected and trials containing blinks or eye movements were discarded. Trials containing muscle activity or jump artifacts due to MEG sensors were discarded using semi-automatic artifact detection routines. Between $3.9 \%$ and $6.8 \%$ of all trials were discarded on average per condition, and the percentage of trials discarded was evenly distributed over conditions. Data were filtered with a $40 \mathrm{~Hz}$ low-pass filter and baseline corrected using a $200 \mathrm{~ms}$ interval prior to the target onset.

For each channel, target locked averages were computed across trials containing square or diamond targets separately for masking and pseudomask conditions and SOAs. In addition, we computed mask locked averages, which provide the opportunity to look at components that are phase locked to the onset of the mask stimulus. Additionally, they allow for the contrast between the effect of the physical difference between the two masks and the effect of visibility. The effect of physical difference should result in a constant difference between VEF over SOAs, while the visibility effect is hypothesized to vary only corresponding to decreased visibility at certain SOAs.

To correct for subjects' head positions relative to the gradiometers, the target and mask locked averages were realigned using a spatial interpolation technique to a template gradiometer array that was constructed from the average position of the subjects relative to the helmet (Knösche, 2002). A grand average over subjects was computed for each SOA in the masking and pseudomask condition and for the target alone and mask alone conditions. To further increase the signal to noise ratio, 
we selected channels in three regions of interest contralateral to the stimulus, and averaged the VEFs within these channel groups. The channels in each region were selected according to the similarity of the VEFs, based on visual inspection. The channels showing opposite polarity to those channels included in the clusters showed opposite field VEFs. Due to the smaller amplitude of the VEFs in these channels compared to the channels in the clusters we did not include these in the analysis. Three channels located over the left occipital cortex formed the occipital cluster, three channels located over the left temporal cortex formed the temporal cluster and five channels overlying the left parietal cortex formed the parietal cluster (see Figure 3d).

For each respective SOA, the amplitude of the VEF in the masking condition was compared to the amplitude of the VEF in the pseudomask condition in both early and late intervals of the VEF. For the target locked averages, the first interval was a window selected around the first VEF component from 60 to $90 \mathrm{~ms}$. The latencies of later intervals were chosen according to intervals shown to reflect masking in earlier studies: The second interval was a window from 190 to $210 \mathrm{~ms}$ (Vaughan \& Silverstein, 1968) and the third interval was taken from 240 to $260 \mathrm{~ms}$ (Bridgeman, 1988). These two late intervals, motivated by earlier EEG studies, fall within a period where mask related activation is present in some conditions, but not in others. To circumvent this possible confound we choose a fourth late interval at a latency from 290 to 390 ms, where mask related activity has decayed in all conditions, and hence would reflect higher cognitive, post perceptual processes. For the mask locked averages, the early interval was selected around the first peak component from 65 to $110 \mathrm{~ms}$. The late interval was a window from 100 to $200 \mathrm{~ms}$ containing the first downward peak in the VEF.

A two-factorial repeated measure ANOVA with variables mask type and SOA was computed for evoked fields averaged over time in each time window and cluster. If stimulus visibility is reflected in VEF amplitude, this should yield an interaction effect between mask type and SOA because the way in which amplitude is modulated across SOAs should be different for the masking and pseudomask condition.

\section{RESULTS}

\section{Behavioral data}

The masking condition produced stronger masking than the pseudomask condition, $F(1,13)=154.3$, $p<.01$. There is a clear $\mathrm{U}$-shaped modulatory effect

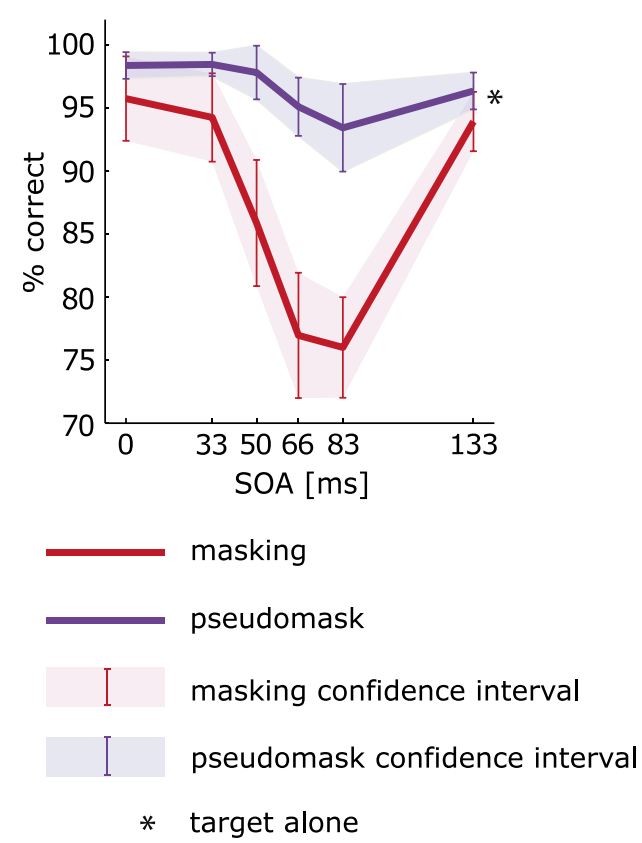

\section{Figure 2. Behavioral data.}

Percent correct scores in the masking condition (red line) and the pseudomask condition (blue line) averaged over all subjects. The error bars as well as the shaded area in the background depict the 95\% confidence interval. There is a significant interaction between mask type and SOA, which is reflected in the U-shaped masking function in the masking condition and a flat masking function in the pseudomask condition.

of SOA on visibility in the masking but not in the pseudomask condition, see Figure 2 . This is reflected by a significant interaction effect between mask type and SOA, $F(5,65)=26.3, p<.01$.

\section{MEG data}

In visual evoked fields, we only found an interaction effect between mask type and SOA in the latest time window, 290-390 ms, in the parietal cluster. For the other time windows, no interaction effect was found in any region of interest, despite the presence of clear visual evoked fields. Both target and mask evoke a clear response in channels overlying the occipital cortex, as can be seen in Figure 3a, 3b, and 3e. The first peak, around $90 \mathrm{~ms}$, reflects target related activity (Figure $3 \mathrm{a}$ and $3 \mathrm{~b}$ ). The amplitude of the peak for SOA 0 is increased relative to the VEF at other SOAs due to the simultaneous presentation of target and mask. The second positive peak in all SOA bins reflects mask related activity. The peak amplitudes shift in latency, corresponding to the shift in onset of the mask at that particular SOA. Figure 3c shows the difference waves between the masking and pseudomask condition for each SOA and the $95 \%$ confidence interval of the dif- 
Table 1.

Significance levels of effects in time intervals and clusters.

\begin{tabular}{|c|c|c|c|c|c|}
\hline Early intervals & & & $60-90 \mathrm{~ms}$ & $65-110 \mathrm{~ms}$ & \\
\hline Cluster & Variable & & $F$ & $F$ & \\
\hline \multirow[t]{3}{*}{ Occipital } & Mask type & $d f(1,13)$ & 0.7 & 2.6 & \\
\hline & SOA & $d f(5,65)$ & $19.7 * *$ & $3.3^{*}$ & \\
\hline & Mask*SOA & $d f(5,65)$ & 1.6 & 1.2 & \\
\hline \multirow[t]{3}{*}{ temporal } & Mask type & $d f(1,13)$ & 0.8 & $4.8^{*}$ & \\
\hline & SOA & $d f(5,65)$ & $9.6 * *$ & $4.2^{* *}$ & \\
\hline & Mask*SOA & $d f(5,65)$ & 1.0 & 0.9 & \\
\hline \multirow[t]{3}{*}{ Parietal } & Mask type & $d f(1,13)$ & 0.0 & $4.2+$ & \\
\hline & SOA & $d f(5,65)$ & $7.9 * *$ & $5.7 * *$ & \\
\hline & Mask*SOA & $d f(5,65)$ & 1.0 & 1.9 & \\
\hline Late intervals & & & $190-210 \mathrm{~ms}$ & $240-260 \mathrm{~ms}$ & $100-200 \mathrm{~ms}$ \\
\hline Cluster & Variable & & $F$ & $F$ & $F$ \\
\hline \multirow[t]{3}{*}{ occipital } & Mask type & $d f(1,13)$ & $11.8^{* *}$ & $5.5^{*}$ & $15.1 * *$ \\
\hline & SOA & $d f(5,65)$ & $4.6 * *$ & $3.2 * *$ & 1.7 \\
\hline & Mask*SOA & $d f(5,65)$ & 1.3 & 0.8 & 0.3 \\
\hline \multirow[t]{3}{*}{ temporal } & Mask type & $d f(1,13)$ & $22.3 * *$ & $6.9 *$ & $13.2 * *$ \\
\hline & SOA & $d f(5,65)$ & $3.8 * *$ & 2.3 & $3.7 * *$ \\
\hline & Mask*SOA & $d f(5,65)$ & 1.45 & 0.7 & 0.9 \\
\hline \multirow[t]{3}{*}{ parietal } & Mask type & $d f(1,13)$ & 2.3 & 0.5 & 1.4 \\
\hline & SOA & $d f(5,65)$ & 2.0 & $5.6 * *$ & $23.0 * *$ \\
\hline & Mask*SOA & $d f(5,65)$ & 2.1 & 0.7 & 0.6 \\
\hline Late intervals & & & $290-390 \mathrm{~ms}$ & & \\
\hline Cluster & Variable & & $F$ & & \\
\hline \multirow[t]{3}{*}{ occipital } & Mask type & $d f(1,13)$ & 0.14 & & \\
\hline & SOA & $d f(5,65)$ & $2.4^{*}$ & & \\
\hline & Mask*SOA & $d f(5,65)$ & $2.3+$ & & \\
\hline \multirow[t]{3}{*}{ temporal } & Mask type & $d f(1,13)$ & 0.7 & & \\
\hline & SOA & $d f(5,65)$ & $2.4^{*}$ & & \\
\hline & Mask*SOA & $d f(5,65)$ & 1.7 & & \\
\hline \multirow[t]{3}{*}{ parietal } & Mask type & $d f(1,13)$ & 0.6 & & \\
\hline & SOA & $d f(5,65)$ & $2.7^{*}$ & & \\
\hline & Mask*SOA & $d f(5,65)$ & $3.9 *$ & & \\
\hline anterior & Mask type & $d f(1,13)$ & 0.04 & & \\
\hline \multirow[t]{2}{*}{ temporal } & SOA & $d f(5,65)$ & $8.4 * *$ & & \\
\hline & Mask*SOA & $d f(5,65)$ & $3.3^{*}$ & & \\
\hline
\end{tabular}

$* p<.05+p=.06$

$* * p<.01$

ference averaged over the whole time epoch, which is plotted in the background. No correction has been performed for multiple comparisons over all latency bins. There seems to be a small difference between the masking and pseudomask condition that shifts with SOA between 100 and 200 ms after mask onset (100-333 ms after target onset). This effect shows up as a main effect of mask type in the mask locked 100-200 ms interval, see Table 1.

\section{Early intervals}

None of the early intervals $(60-90 \mathrm{~ms}$ and 65$-110 \mathrm{~ms}$ ) show a modulation of VEF amplitude related to target visibility. This is reflected by the absence of inter- 

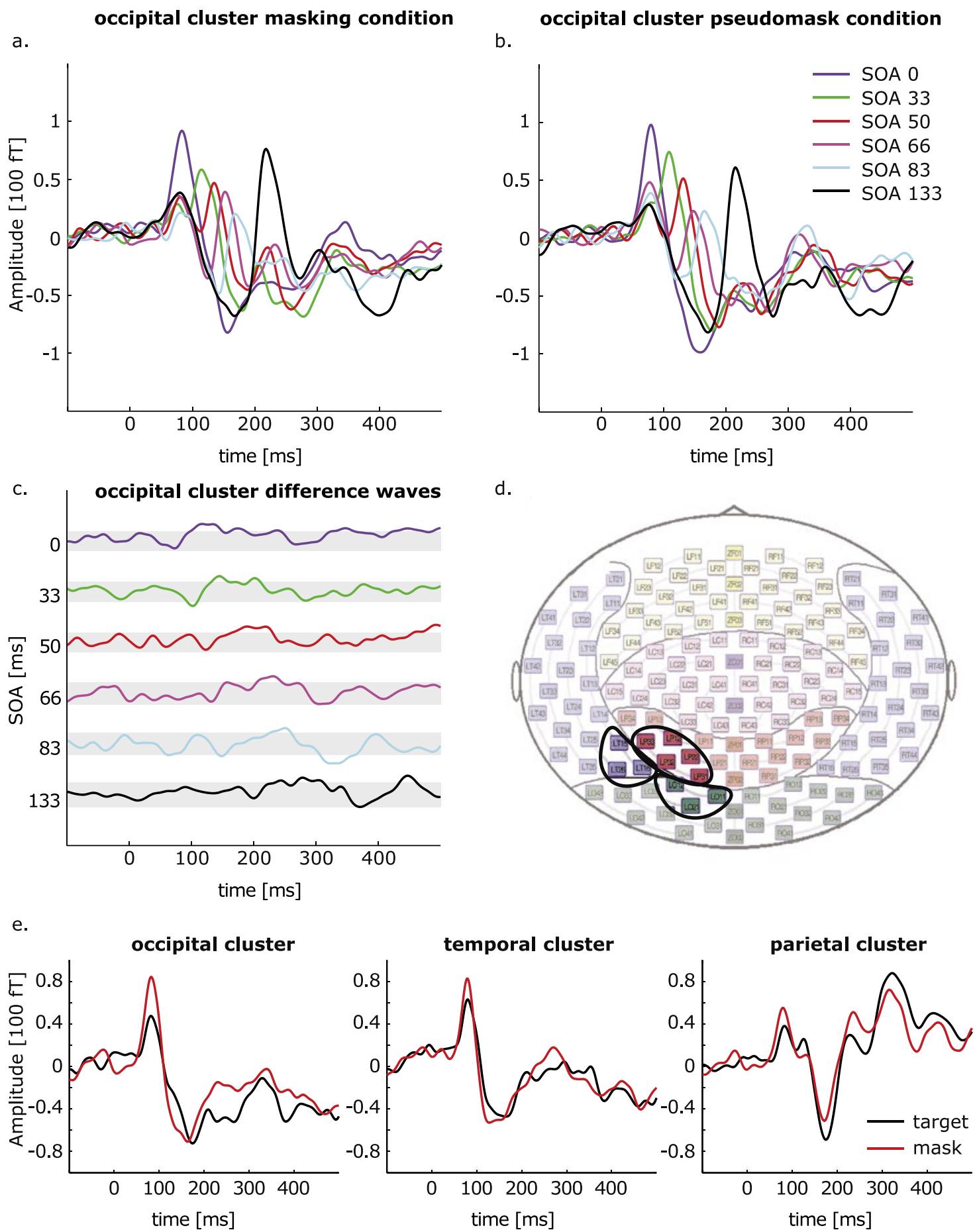

\section{Figure 3. VEF of target locked avgerages.}

Target locked VEFs. a. VEFS in the masking condition for each SOA of the channel cluster overlying occipital cortex. There is a clear VEF to the target as well as a VEF to the mask. The latency of the mask related peak shifts with the onset shift of the mask at different SOAs. b. VEFs in the pseudomask condition of the same cluster. These VEFs show the same pattern as the masking condition c. Difference waves for each SOA between masking condition and pseudomask condition of the same cluster. The shaded areas in the background are the 95\% confidence intervals averaged over the whole epoch (no multiple comparison correction has been performed). There is no clear difference between the masking and pseudomask condition which could be related to target visibility. There seems to be a small difference that shifts with SOA (thus being mask locked) between 100 and 200 ms after mask onset (100-333 ms after target onset). This difference is reflected in the significant effect of mask type in the 100-200 ms interval of the mask locked averages, see table 1. d. Topographical layout of the 151 MEG sensors. The location of the occipital (green), the temporal (blue), and the parietal (red) cluster are highlighted. e. VEFs of the target alone conditions (black line) and the mask alone conditions (red line) for all three clusters. 

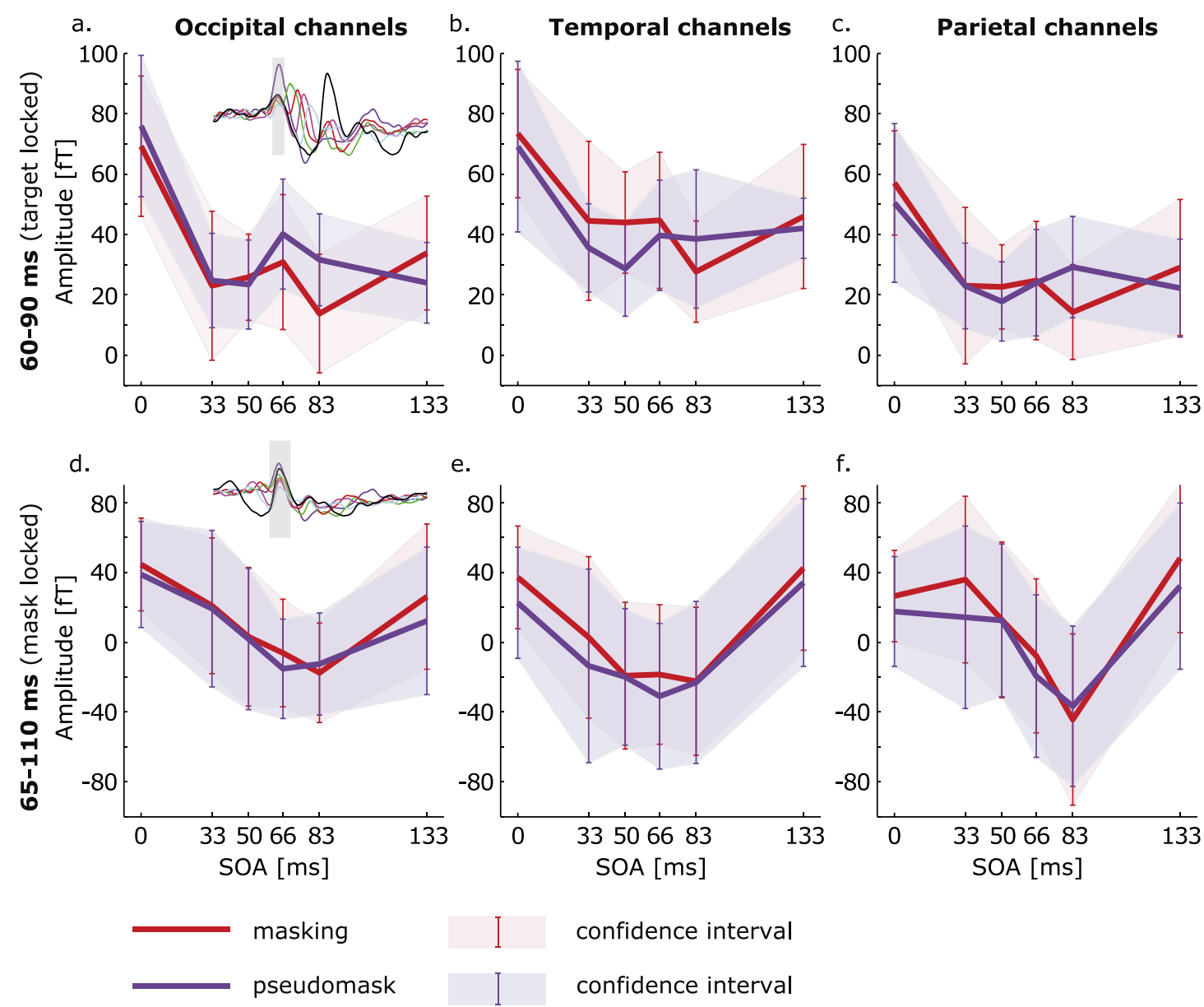

confidence interval

confidence interval

Figure 4. VEF amplitude in the early intervals for target and mask locked data.

Amplitude averaged over a time interval encompassing the early VEFs of the three clusters for both the masking condition (red line) and the pseudomask condition (blue line). a. Occipital cluster, target locked. b. Temporal cluster, target locked, c. Parietal cluster, target locked, d. Occipital cluster, mask locked, e. Temporal cluster, mask locked, f. Parietal cluster, mask locked.

None of the time intervals or clusters shows a significant interaction effect between mask type and SOA.

actions between mask type and SOA in the target locked components [occipital: $F(5,65)=1.6, p=.2$, temporal: $F(5,65)=1.0, p=.4$, parietal: $F(5,65)=1.0$, $p=.4]$ and in the mask locked components for all clusters [occipital: $F(5,65)=1.2, p=.3$, temporal: $F(5,65)=0.9, p=.5$, parietal: $F(5,65)=1.9, p=.1]$. The statistics are summarized in Table 1 . Figure 4 shows VEF amplitudes averaged over a time window around the first visible peak for each SOA in both the masking and pseudomask condition.

The amplitudes of both the target and mask locked averages are modulated by SOA. Figures $4 a$ to $4 \mathrm{c}$ show this main effect of SOA in the target locked data for all channel clusters [occipital: $F(5,65)=19.7$, $p<.01$, temporal: $F(5,65)=9.6, p<.01$, parietal: $F(5,65)=7.9, p<.01]$. This effect is due to the increased amplitude with the simultaneous presentation of target and mask at SOA 0 . Figures $4 \mathrm{~d}$ to $4 \mathrm{f}$ show the effect of SOA in the mask locked data [occipital: $F(5,65)=3.3, p=.01$, temporal: $F(5,65)=4.2$, $p<.01$, parietal: $F(5,65)=5.7, p<.01]$. The effect of mask type is only significant in the mask locked data for the channels overlying the temporal cortex $[F(1,13)=4.8, p=.05]$ and not in the target locked data, see Table 1 .

\section{Later intervals}

The masking and pseudomask condition did not produce different modulations of VEF amplitude related to visibility during the $190-210 \mathrm{~ms}$ and the $240-$ $-260 \mathrm{~ms}$ interval in the target locked data, or during the 100-200 ms interval in the mask locked data. Figures $5 a$ to $5 c$ show the averaged amplitudes over the target locked time window from 190-210 ms for all channel groups. There are no significant interaction effects in this interval [occipital: $F(5,65)=1.3$, 

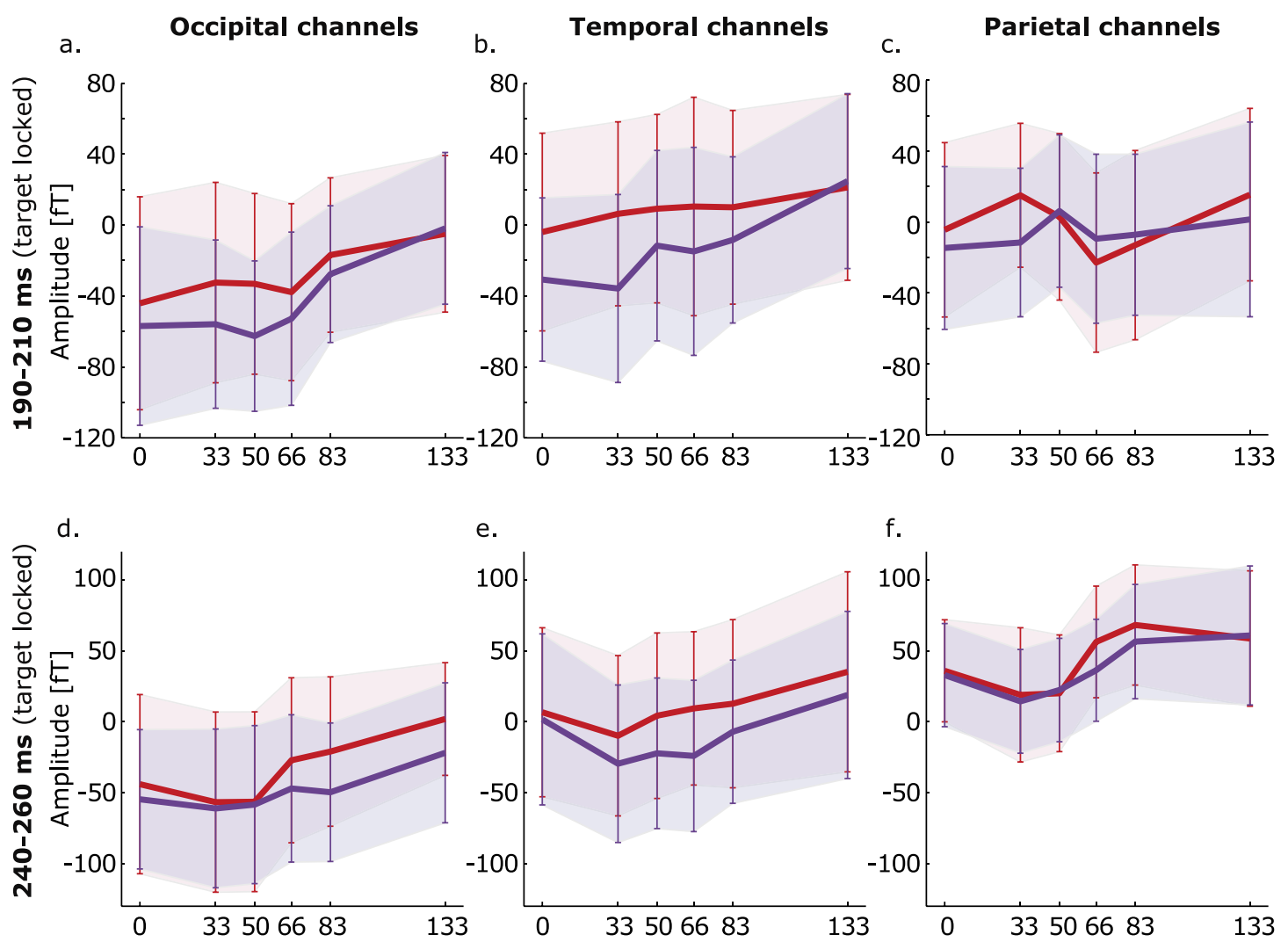

g.

h.
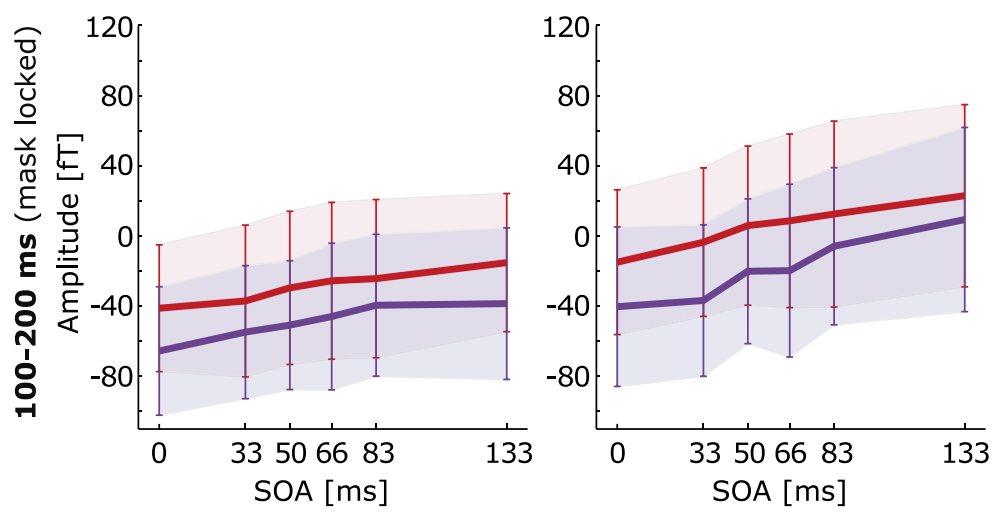

i.

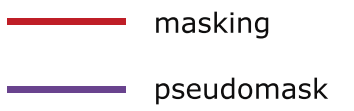

confidence interval

confidence interval

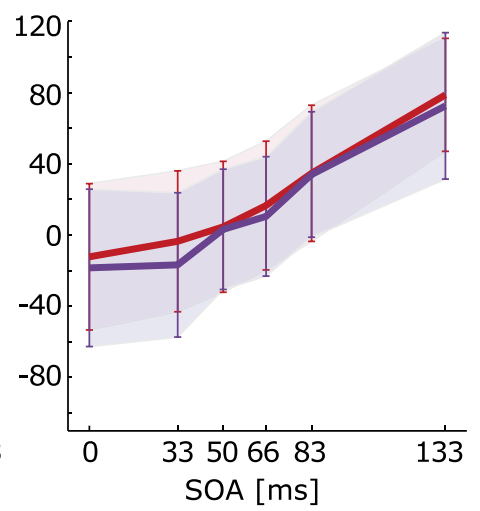

Figure 5. VEF amplitude in the 190 to $210 \mathrm{~ms}$ and 240 to $260 \mathrm{~ms}$ intervals for target and mask locked data.

Amplitude averaged over later time intervals of the three clusters for both the masking condition (red line) and the pseudomask condition (blue line). a./d. Occipital cluster, target locked. b./e. Temporal cluster, target locked, c./f. Parietal cluster, target locked, g. Occipital cluster, mask locked, h. Temporal cluster, mask locked, i. Parietal cluster, mask locked. None of the time intervals or clusters shows a significant interaction effect between mask type and SOA.

$p=.3$, temporal: $F(5,65)=1.45, p=.2$, parietal: $F(5,65)=2.1, p=.08]$. However, for both the occipital and the temporal channels there is a main effect of both mask type and SOA [occipital: mask type: $F(1,13)=11.8, p<.01$, SOA: $F(5,65)=4.6$, $p<.01$, temporal: mask type: $F(1,13)=22.3$, $p<.01$, SOA: $F(5,65)=3.8, p<.01$, parietal: mask type: $F(1,13)=2.3, p=.2$, SOA: $F(5,65)=2.0$, $p=.09]$. Figures $5 \mathrm{~d}$ to $5 f$ show the averaged amplitudes over the target locked time window from 240- 
$-260 \mathrm{~ms}$. There is also no interaction effect in this interval for any of the clusters [occipital: $F(5,65)=0.8$, $p=.5$, temporal: $F(5,65)=0.7, p=.6$, parietal: $F(5,65)=0.7, p=.6]$. There is a main effect of SOA in the occipital and parietal channels and a trend is seen in the temporal channels [occipital: $F(5,65)=3.2$, $p<.01$, temporal: $F(5,65)=2.3, p=.06$, parietal: $F(5,65)=5.6, p<.01]$. A main effect of mask type is shown in the occipital and temporal channels [occipital: $F(1,13)=5.5, p=.04$, temporal: $F(1,13)=6.9$, $p=.02$, parietal: $F(1,13)=0.5, p=.5]$. Figures $5 g$ to $5 i$ depict the averaged amplitude during the later time window of the mask locked averages. We also did not find significant interaction effects in this late interval of the mask locked average [occipital: $F(5,65)=0.3$, $p=.9$, temporal: $F(5,65)=0.9, p=.5$, parietal: $F(5,65)=0.6, p=.7]$. The occipital and temporal channels show an effect of mask type [occipital: $F(1,13)=15.1, p<.01$, temporal: $F(1,13)=13.2$, $p<.01$, parietal: $F(1,13)=1.4, p=.3]$. The temporal and parietal channels show an effect of SOA [occipital: $F(5,65)=1.7, p=.1$, temporal: $F(5,65)=3.7$, $p<.01$, parietal: $F(5,65)=23, p<.01]$. This modulation is not even slightly $U$-shaped as in the target locked averages, but increases monotonically with SOA: the closer the target stimulus is to the mask in time, the more influence it has on the first downward component of the VEF.

Because the amplitudes of the VEF at each SOA in the 190-210 ms and $240-260 \mathrm{~ms}$ intervals do not show a very clear U-shape function, which we expected looking at Bridgeman's data (1988), we computed a Pearson correlation coefficient for broader time windows in the target locked data. Finding a significant correlation between the behavioral masking function and VEF amplitude of both the masking and pseudomask condition would show that a reported $\mathrm{U}$-shape is not necessarily due to decreased visibility, but that it could be due to the temporal overlap between target and mask stimuli. We found significant correlations between the behavioral masking function and VEF amplitudes at different time intervals. During an interval between 130-180 ms, in a cluster of channels overlapping with the left occipital cluster, the correlation coefficient is close to significance for the masking condition $[r(6)=-0.76, p=.079]$, and is significant for the pseudomask condition $[r(6)=-0.90$, $p=.015]$, see Figure 6 . For a later interval of $250-$ $-300 \mathrm{~ms}$ both the masking and pseudomask condition correlate significantly with the behavioral masking function in a cluster of channels overlapping with the temporal cluster, Figure $3 d$ [masking con-

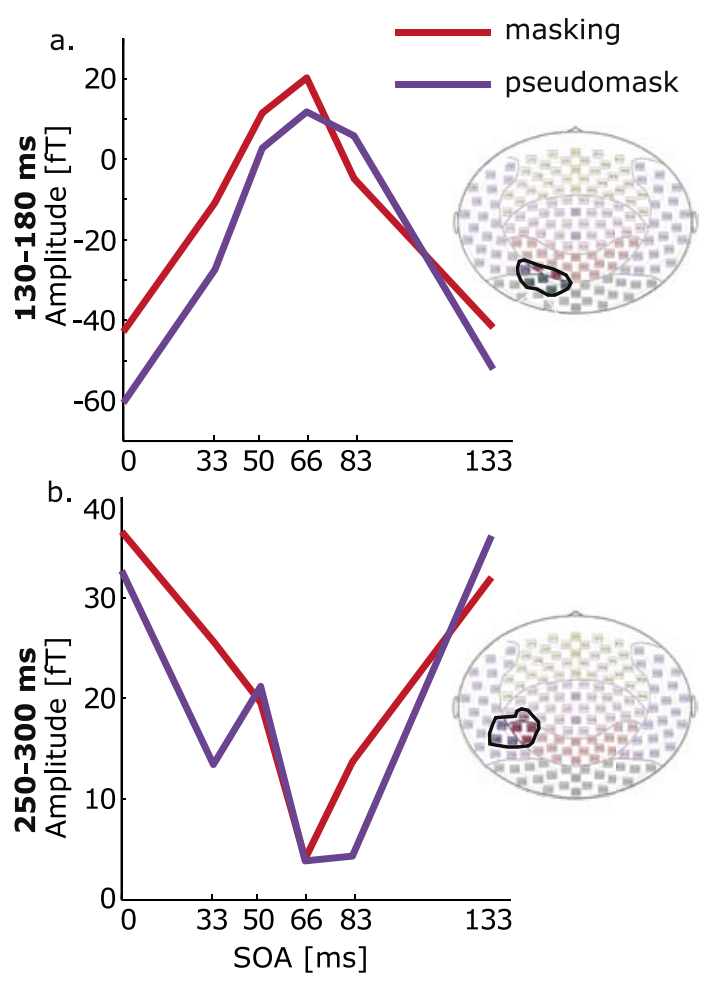

Figure 6. Correlation coefficients of the VEF amplitude in the 130 to $180 \mathrm{~ms}$ and 250 to $300 \mathrm{~ms}$ interval with the behavioral masking function.

Amplitude averaged over later time intervals of two clusters for both the masking condition (red line) and the pseudomask condition (blue line). a. 130-180 ms interval at a occipital cluster shown in the topographical plot to the right. b. 250-300 ms interval at a temporal cluster shown in the topographical plot to the right. Both the masking and the pseudomask condition show significant or nearly significant correlations with the behavioral masking function.

dition, $r(6)=0.93, p=.008$, pseudomask condition $r(6)=0.82, p=.045]$.

However, during a later time window between 290 and $390 \mathrm{~ms}$, the masking and pseudomask condition yield different modulations of VEF amplitude in the parietal cluster. This is indicated by a significant interaction effect of SOA and mask type on VEF amplitude, see Figure $7 a[F(5,65)=3.9, p=.004]$. The occipital and the temporal cluster do not show this effect in this time interval [occipital: $F(5,65)=2.3, p=.052$, temporal: $F(5,65)=1.7, p=.14]$. In all three clusters we see a main effect of SOA [occipital: $F(5,65)=2.4$, $p=.05$, temporal: $F(5,65)=2.4, p=.048$, parietal: $F(5,65)=2.7, p=.03$ ], but not of mask type [occipital: $F(1,13)=0.1, p=.7$, temporal: $F(1,13)=0.7, p=.4$, parietal: $F(1,13)=0.6, p=.4$ ]. The topographical distribution of the interaction effect in this time interval, calculated by subtracting the average of the SOA 50, 66, and 83 trials in the masking condition from the corre- 

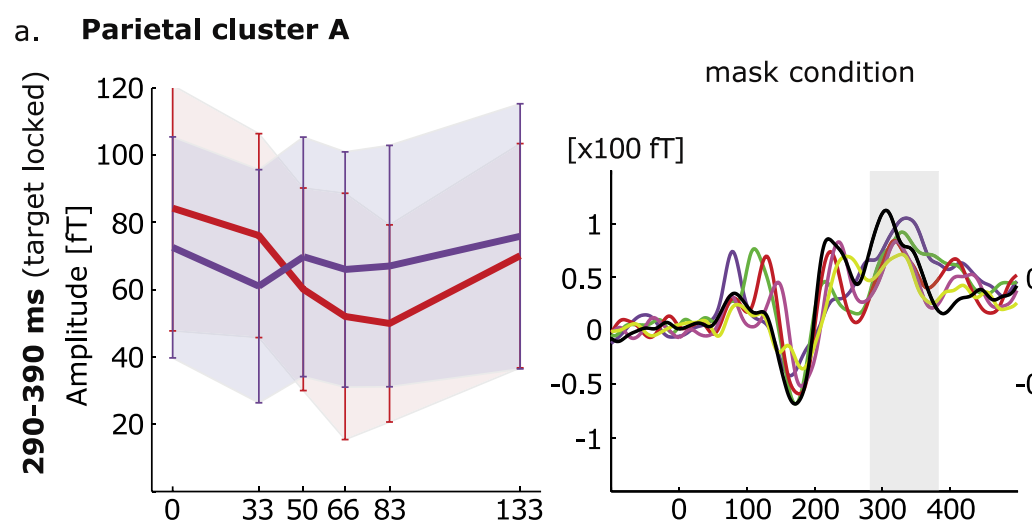

pseudomask condition

\section{b. Temporal cluster B}
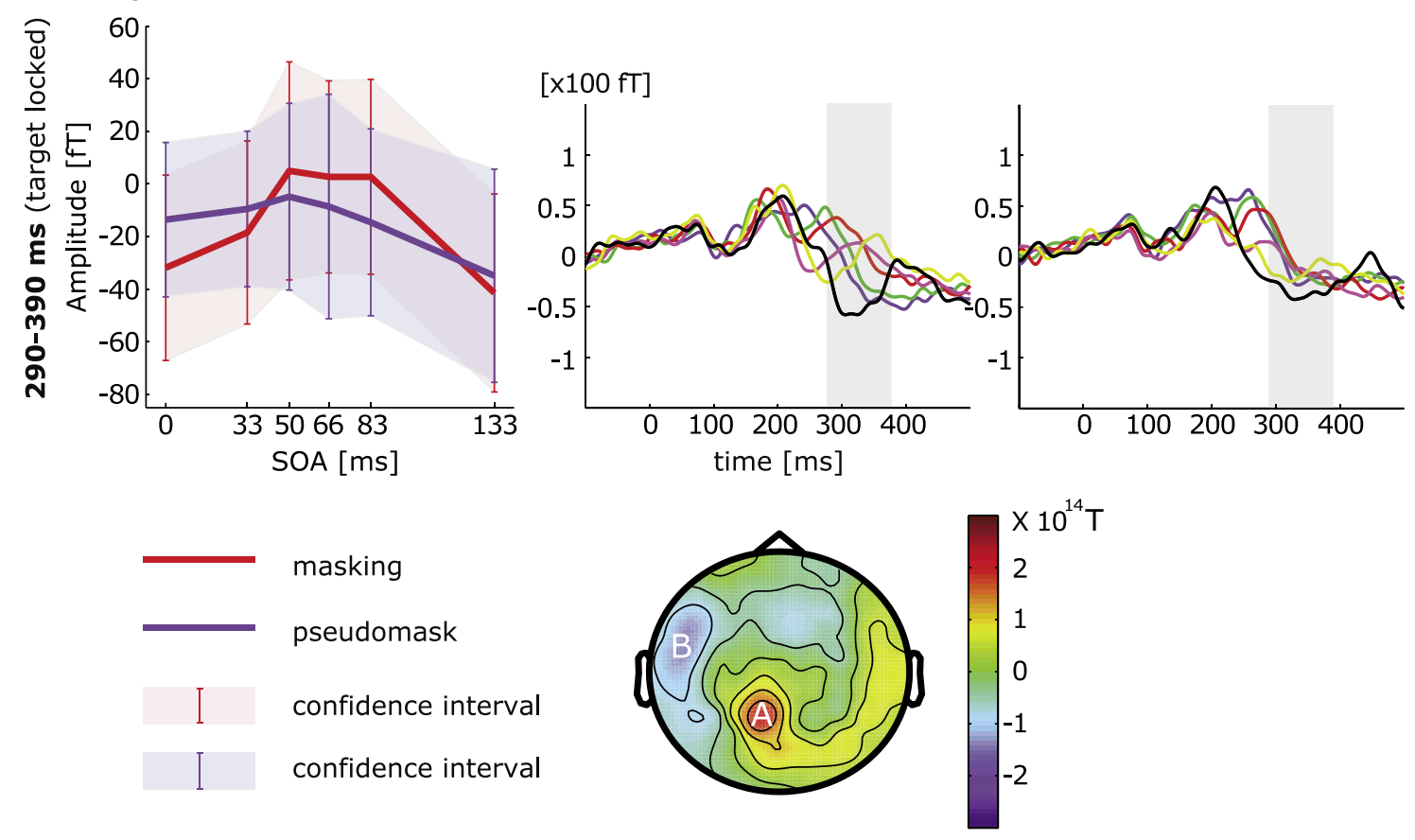

Figure 7. Interaction effect in the $\mathbf{2 9 0}$ to $\mathbf{3 9 0} \mathbf{~ m s ~ i n t e r v a l ~ f o r ~ t a r g e t ~ l o c k e d ~ d a t a . ~}$

Amplitude averaged over a time interval from 290 to $390 \mathrm{~ms}$ for both the masking condition (red line) and the pseudomask condition (blue line). a. VEF amplitudes of cluster A (see Figure 7c) showing an interaction effect between SOA and mask type. The masking condition does correlate with the behavioral masking function while the pseudomask condition does not. This graph is flanked by the VEFs from the masking and pseudomask condition of the same cluster. The gray bar indicates the time interval. b. VEF amplitudes of cluster B showing an interaction effect between SOA and mask type. The masking condition does correlate with the behavioral masking function while the pseudomask condition does not. Also the corresponding VEFS of the masking and pseudomask condition of the same cluster are shown. The shape of the amplitude curve shows an opposite polarity pattern compared to the parietal cluster A. c. Topographical plot of the difference between the average of the SOA 50, 66, and 83 trials in the masking condition and the corresponding SOA trials in the pseudomask condition averaged over the 290 to 390 ms interval.

sponding trials of the pseudomask condition, displays an additional cluster of three more anterior temporal channels, see Figure 7c. The averaged VEFs over these channels in this time interval also show the interaction effect between mask type and SOA $[F(5,65)=3.3, p=.01]$ and a main effect of $\operatorname{SOA}[F(5,65)=8.4, p<.001]$, but no effect of mask type $[F(1,13)=.04, p=.8]$. Figure $7 \mathrm{~b}$ shows that the polarity of the $\mathrm{U}$-shaped effect is opposite in sign to the effect seen in the parietal cluster.
In addition to this significant interaction effect, we see a correlation between the behavioral masking function and the masking condition in this postperceptual interval, in both the parietal and the more anterior temporal cluster [parietal: $r(6)=0.96$, $p=.003$, anterior temporal: $r(6)=-0.84, p=.037]$. But, there is no correlation between the pseudomask condition and the behavioral masking data in the same clusters [parietal: $r(6)=0.308, p=.55$, 
anterior temporal: $r(6)=-0.35, p=.5]$, see Figure 7.

\section{DISCUSSION}

The aim of this study was to investigate whether modulations of early or late VEFs can be regarded as neurophysiological correlates of metacontrast masking. We compared behavioral data and evoked brain responses to masked and non masked target stimuli over a range of SOAs from 0 to $133 \mathrm{~ms}$. Visibility followed a $\mathrm{U}$-shaped masking function in the masking condition, with the trough at 66 and 83 ms, while staying above $90 \%$ at all SOAs in the pseudomask condition. This was reflected in a significant interaction effect between the type of mask and SOA.

The analysis of the visual evoked fields focused on finding an interaction effect in early and late VEFs in both target and mask locked averages comparable to the effect seen in the behavioral data. This interaction effect, indicating a modulation of VEF amplitude over SOA in the masking condition, but not in the pseudomask condition, would reflect a neural correlate of stimulus visibility.

In the early timewindows we found that the evoked response to an initial stimulus is modulated by a second stimulus presented in close temporal succession, even though this modulation is unrelated to the visibility of the first stimulus. A differential response to the two mask stimuli was observed only in the mask locked averages of the cluster overlying temporal cortex. We interpret this as reflecting a difference in the processing of the physical properties of the two masks. Previous EEG studies also failed to find any modulation of early visual evoked components by visual masking (Bridgeman, 1988; Jeffreys \& Musselwhite, 1986; Schiller \& Chorover, 1966; Vaughan \& Silverstein, 1968). Given the present and previous results, it seems unlikely that the mechanism leading to the masking effect manifests itself in the first neural response to the stimulus.

Studies by Bridgeman (1988) and Vaughan \& Silverstein (1968) suggest that the modulatory effect of visibility should manifest itself in later timewindows. Therefore, we analyzed additional timewindows of 190 to $210 \mathrm{~ms}$ and 240 to $260 \mathrm{~ms}$ for the target locked averages, and a window between 100 and $200 \mathrm{~ms}$ for the mask locked averages. If backward masking leads to disruption of recurrent target activity, a modulation that reflects behavioral changes is expected at these latencies (Bridgeman, 1988; Vaughan \& Silverstein, 1968).

In none of these intervals did we find an interaction effect reflecting the visibility of the target stimulus.
However, we observed effects of SOA and mask type on several of the channel clusters, or intervals. In target locked averages on channels overlying occipital cortex, we found that SOA influences VEF amplitude, reflecting the different temporal overlap between the target and mask related activity at different SOAs. This effect of SOA on amplitude was obliterated by averaging data locked to the mask. Both the target and mask locked averages in these three intervals reflected differential perceptual processing of the two different mask stimuli shown by an effect of mask type. Thus, these late intervals on the occipital channels reflect the type of stimuli and their temporal organization, but show no modulation related to visibility.

The same results also hold for the channels overlying part of temporal cortex. There is no effect of target visibility, but the data reflect the temporal overlap between target and mask processing and the differential response to the two masks. Also the channels overlying part of parietal cortex fail to show an effect of visibility. They show an effect of the temporal overlap of the stimuli in the 240-260 ms target locked interval and in the 100-200 ms mask locked interval, but do not show an effect of mask type.

In addition to these later intervals (190 to $210 \mathrm{~ms}$ and 240 to $260 \mathrm{~ms}$ ), a postperceptual time interval in the target locked averages was analyzed. Unlike in the two earlier intervals, the parietal cluster in the 290-390 ms interval shows an interaction effect between mask type and SOA. VEFs in the masking condition were deflected more strongly than those in the pseudomask condition. Further, only VEF amplitude in the masking condition showed a significant correlation with the behavioral data. This suggests that the VEF in this time interval reflects the visibility of the target stimulus. In addition, a main effect of SOA in all clusters was found. There was no effect of mask type, which indicates that the evoked response in this 290-390 ms interval is unaffected by differences in physical stimulation. Thus, this interval most likely reflects postperceptual processing. Bridgeman's (1988) observation of the effect of visibility is at a latency most likely to be perceptual in origin. Therefore, we can conclude that the effect we see is not comparable to Bridgeman's (1988) observations of the effect of visibility. The activities of both effects reflect different stages in visual processing. Furthermore, the absence of a main effect of mask type also shows that the behavioral performance is a genuine masking effect and not due to better target recovery in the pseudomask condition because of, for example, visible figural changes of the pseudomask that are produced by the 
target. If this were the case then one should also see an effect of mask type in this cluster.

An additional cluster of channels overlying the temporal cortex shows the interaction effect in opposite polarity. This bipolar pattern over temporal and parietal cortex suggests a source lying in the temporal-parietal cortex. However, source modeling of late components usually involves multiple sources. Our single-subject data were not of sufficient quality to perform individual source analyses. In the grand averaged data, the interindividual differences between orientation and location of the individual sources cause a spatial blurring of the VEF topography, making it difficult to determine the appropriate number of sources required for the grand average, and thus leading to a potential mislocalization of the sources. Therefore we refrained from source analysis.

This later component from 290 to 390 ms, which could reflect target visibility, can be compared to the P300 component in ERP research. It is assumed that the P300 reflects a process related to attention and working memory (Kok, 2001; Vogel, Luck, \& Shapiro, 1998). Several attentional blink studies have shown that the P300 is suppressed or absent when a second target is not detected, compared to a present P300 when the second target is detected (Kranczioch, Debener, \& Engel, 2003; Rolke et al., 2001). It is concluded in these studies that the second target does reach the point of perceptual representation, but is not consolidated for subsequent report. This hypothesis can also be applied to the mechanism of metacontrast masking. We found a suppression of the amplitude of the VEF in those conditions showing the strongest masking, that is, SOA 50, 66, and 83 compared to those conditions in which the target was clearly visible. Assuming that this component is comparable to the P300, we argue that the masking of the target has taken place before the stimulus has formed a stable representation in working memory. There are several explanations for this failure to form a stable representation (Kranczioch et al., 2003). First, the information of the target is not successfully transferred into working memory. Second, the information is not selected by an attentional mechanism to enter working memory. Or third, it has failed to match with a template which is held in working memory.

This finding points to the conclusion that metacontrast masking is not due to a disruption of object identification, but rather occurs at a postperceptual phase when object related information is selected for, or enters, working memory. This account of metacontrast masking fits in with a broad range of priming studies in which a stimulus that is not consciously perceived nevertheless influences later behavior (Vorberg, Mattler, Heinecke, Schmidt, \& Schwarzbach, 2003).

In contrast to the findings of Bridgeman (1988), the present data do not show a U-shaped modulation in the VEF amplitude at the 190-210 ms and 240$-260 \mathrm{~ms}$ interval. However, when looking at broader time intervals we found significant correlations between the behavioral $U$-shaped masking function and the VEF amplitude over SOA in the masking condition of the MEG data. More interesting is the fact that this correlation is also present in the pseudomask condition. The VEF amplitude decreases at those SOAs where masking is strongest. This indicates that the overlap between target and mask related activity results in a U-shaped modulation of VEF amplitude that seems similar, but is unrelated to, the visibility of the target stimulus. Even though the latencies at which we found these correlations are not directly comparable to the latency at which Bridgeman reports seeing a U-shape, we do want to question Bridgeman's interpretation that the $\mathrm{U}$-shaped modulation he observed is a concomitant of metacontrast masking. The U-shaped modulation he observed could also be explained by the temporal overlap between the VEF of the target and the mask stimulus.

Our results do not seem to fit in with other earlier metacontrast masking studies using EEG. Vaughan and Silverstein (1968) compared the VEP of a condition in which masking occurred with the VEP of a comparable condition in which no masking occurred, and showed a reduction in size of the VEP component at $200 \mathrm{~ms}$ after target onset. But, as with the interpretation by Bridgeman (1988), it is unclear whether this reduction reflects behavioral performance, or if it is the result of different temporal overlap between target and mask related activity at different SOAs.

A study which did use a control condition to check for temporal overlap was conducted by Andreassi, De Simone, and Mellers in 1976. They contrasted the modulation of the VEP response for a target presented alone, an effective mask, and an ineffective mask. The attenuation of VEP amplitude with target alone versus effective mask was larger than the attenuation with target alone versus ineffective mask. They concluded that the attenuated VEP reflects visual masking. However, their data raise two questions. In the first place, the mask and nonmasking stimuli produce different VEPs. In the first condition, the data clearly show a target related peak and a second mask related peak that evolves with increasing SOA. This is also shown in Jeffreys and Musselwhite's (1986) data. In 
the nonmasking condition there is only one broad peak present, although it contains masks at the same latencies. Secondly, they present a second mask following the first one. In both the masking and non-masking condition this second mask effectively decreases visibility of the first mask (Breitmeyer, Rudd, \& Dunn, 1981). Following the reasoning of Andreassi et al. (1976), this should result in an attenuation of the VEP related to the first mask, which they did not observe.

In our view these EEG studies do not conclusively show a correlate of metacontrast masking. We believe that the modulation that Bridgeman (1988) and Vaughan and Silverstein (1968) observed is only due to temporal interaction between the processing of the stimuli, and is not a concomitant of stimulus visibility. The conclusions drawn by Andreassi et al. (1976) are debatable considering the aforementioned data issues. The present experiment has shown data that reflect the processing of the stimuli accurately with a high signal to noise ratio. Nevertheless, we observed no differential modulations between masking and non-masking conditions in time intervals around 200 and $250 \mathrm{~ms}$. Therefore, we conclude that the observed $\mathrm{U}$-shaped modulations shown by these studies do not reflect stimulus visibility.

Other studies investigating object recognition with fMRI or cell recordings in monkeys also found changes in neural activity related to whether or not an object was recognized (Bar, et al., 2001; Grill-Spector, Kushnir, Hendler, \& Malach, 2000; Kovacs, Vogels, \& Orban, 1995; Rolls, Tovee, \& Panzeri, 1999). However, these studies used pattern masking in which the target and the mask stimuli are presented at the same retinal location and activate the same part of visual cortex. This paradigm yields a monotonically increasing masking function and is not comparable to metacontrast masking.

A recent study which did use a metacontrast masking paradigm showed that fMRI activity in early visual cortex did not reflect the visibility of a stimulus, but that activity in later visual areas and parieto-frontal regions are correlated with the visibility function (Haynes, Driver, \& Rees, 2005). We believe that the present results are consistent with these findings. Our results show a lack of correlation between early latency MEG components and the masking function, which indicates that the early visual areas are not involved in the masking mechanism, a finding which is comparable to the results of Haynes et al. (2005). We do see a correlation between visibility and a later VEF component on channels overlying the parietal cortex, which is consistent with the finding that fMRI activity in parieto-frontal regions correlates with the visibility of a stimulus.
In addition to expanding the time range of interest in order to include postperceptual processing latencies, future studies investigating metacontrast masking might focus on a different aspect of brain activity. When computing stimulus locked averages, one assumes that the stimulus evokes an effect at a specific latency with the same phase (or polarity) in every trial. All other processes are considered noise and are averaged out. However, several studies have shown that brain activity related to visual awareness is not phase locked, but that it is induced. It is time locked to the stimulus, but with a phase that varies over trials (Engel \& Singer, 2001; Tallon-Baudry \& Betrand, 1999). This type of activity can be revealed through a time-frequency analysis of the data. For metacontrast masking studies this means that the visibility of the target stimulus would be reflected in a modulation of the power in a specific frequency band. Studies on the role of oscillations in visual perception have shown that synchronous oscillations are important in the processes of selective attention and top-down modulation (Engel, Fries, \& Singer, 2001; Fries, Reynolds, Rorie, \& Desimone, 2001). If the mechanism of metacontrast masking is mediated by higher perceptual processes, like feedback or recurrent processes and attentional processes as suggested in several studies (Enns \& Di Lollo, 2000; Ro, Breitmeyer, Burton, Singhal, \& Lane, 2003), then designing metacontrast masking experiments suitable for time-frequency analysis could be an option for future masking studies.

\section{Acknowledgements}

We would like to thank Roel Willems and Mark Rijpkema for valuable input to the manuscript. We also would like to thank the editor and the anonymous reviewers for the constructive and helpful contributions.

\section{References}

Alpern, M. (1953). Metacontrast. Journal of the Optical Society of America, 43, 648-657. [www

Andreassi, J., De Simone, J., \& Mellers, B. (1976). Amplitude changes in the visual evoked cortical potential with backward masking. Electroencephalography and Clinical Neurophysiology, 41, 387-398. [ww]

Bar, M., Tootell, R. B., Schacter, D. L., Greve, D. N., Fischl, B., Medola, J. D., Rosen, B. R., \& Dale, A. M. (2001). Cortical mechanisms specific to explicit visual object recognition. Neuron, 29, 529-535. Www

Breitmeyer, B. (1984). Visual masking: an integrative approach. New York, NY: Oxford University Press. 
Breitmeyer, B., \& Ogmen, H. (2000). Recent models and findings in visual backward masking: a comparison, review, and update. Perception and Psychophysics, 62, 1572-1595. |WwW

Breitmeyer, B., Rudd, M., \& Dunn, K. (1981). Metacontrast investigations of sustained-transient channel inhibitory interactions. Journal of Experimental Psychology: Human Perception and Performance, 7, 770-779. |www

Bridgeman, B. (1975). Correlates of metacontrast in single cells of the cat visual system. Vision Research, 15, 91-99.

Bridgeman, B. (1980). Temporal response characteristics of cells in monkey striate cortex measured with metacontrast masking and brightness discrimination. Brain Research, 196, 347-364. www

Bridgeman, B. (1988). Visual evoked potentials: concomitants of metacontrast in late components. Perception \& Psychophysics, 43, 401-403. [www

Engel, A., Fries, P., \& Singer, W. (2001). Dynamic predictions: oscillations and synchrony in top-down processing. Nature Reviews, Neuroscience, 2, 704-716. $\underline{\omega W w}$

Engel, A., \& Singer, W. (2001). Temporal binding and the neural correlates of sensory awareness. Trends in Cognitive Sciences, 5, 16-25. |WwW

Enns, J., \& Di Lollo, V. (2000). What's new in visual masking? Trends in Cognitive Sciences, 4, 345-352. WWw

Fries, P., Reynolds, J. H., Rorie, A. E., \& Desimone, R. (2001). Modulation of oscillatory neuronal synchronization by selective visual attention. Science, 291, 1560-1563. $\mid \overline{w w \mid}$

Grill-Spector, K., Kusnhir, T., Hendler, T., \& Malach, R. (2000). The dynamics of object-selective activation correlate with recognition performance in humans. Nature Neuroscience, 3, 837-843. Www

Haynes, J., Driver, J., \& Rees, G. (2005). Visibility reflects dynamic changes of effective connectivity between $\mathrm{V} 1$ and fusiform cortex. Neuron, 46, 811-821. |wWw

Jeffreys, D. A. (1971). Cortical source locations of pattern-related visual evoked potentials recorded from the human scalp. Nature, 229, 502-504. |WwW

Jeffreys, D. A., \& Axford, J. G. (1972). Source locations of pattern-specific components of human visual evoked potentials. I. Component of striate cortical origin. Experimental Brain Research, 16, 1-21. |WWw

Jeffreys, D. A., \& Musselwhite, M. J. (1986). A visual evoked potential study of metacontrast masking. Vision Research, 26, 631-642. | $\mid \mathrm{ww}$

Knösche, T. R. (2002). Transformation of whole-head MEG recordings between different sensor positions.
Biomedizinische Technik. Biomedical engineering, 47, 59-62. |www

Kok, A. (2001). On the utility of P3 amplitude as a measure of processing capacity. Psychophysiology, 38, 557-577. Www

Kovacs, G., Vogels, R., \& Orban, G. A. (1995). Cortical correlate of pattern backward masking. Proceedings of the National Academy of Sciences USA, 92, 5587-5591. WwW

Kranczioch, C., Debener, S., \& Engel, A. K. (2003). Event-related potential correlates of the attentional blink phenomenon. Cognitive Brain Research, 17, 177-187.

Lachter, J., Durgin, F. H., \& Washington, T. (2000). Disappearing percepts: evidence for retention failure in metacontrast masking. Visual Cognition, 7, 269-279.

Lamme, V. A. F., \& Roelfsema, P. R. (2000). The distinct modes of vision offered by feedforward and recurrent processing. Trends in Neuroscience, 23, 571-579. WWW

Ro, T., Breitmeyer, B., Burton, P., Singhal, N., \& Lane, D. (2003). Feedback contributions to visual awareness in human occipital cortex. Current Biology, 11, 1038-1041. [WW

Rolke, B., Heil, M., Streb, J., \& Hennighausen, E. (2001). Missed prime words within the attentional blink evoke an N400 semantic priming effect. Psychophysiology, 38, 165-174. |www

Rolls, E. T., Tovee, M. J., \& Panzeri, S. (1999). The neurophysiology of backward visual masking: information analysis. Journal of Cognitive Neuroscience, 11 , 300-311. WwW

Schiller, P., \& Chorover, L. (1966). Metacontrast: its relation to evoked potentials. Science, 153, 13981400. $\underline{W w W}$

Tallon-Baudry, C., \& Betrand, O. (1999). Oscillatory gamma activity in humans and its role in object representation. Trends in Cognitive Sciences, 3, 151-162. WWW

Vaughan, H., \& Silverstein, L. (1968). Metacontrast and evoked potentials: a reappraisal. Science, 160, 207-208. WWW

Vogel, E. K., Luck, S. J., \& Shapiro, K. L. (1998). Electrophysiological evidence for a postperceptual locus of suppression during the attentional blink. Journal of Experimental Psychology: Human Perception and Performance, 24, 1656-1674. Www

Vorberg, D., Mattler, U., Heinecke, A., Schmidt, T., \& Schwarzbach, J. (2003). Different time courses for visual perception and action priming. Proceedings of National Academy of Sciences, 100, 6275-6280. Www 